\title{
Parametric estimation of ultrasonic phase velocity and attenuation in dispersive media
}

\author{
Jesper Martinsson *, Johan E. Carlson \\ EISLAB, Luleå University of Technology, SE-971 87 Luleå, Sweden
}

Available online 5 June 2006

\begin{abstract}
In ultrasonic characterization of liquids, gases, and solids, accurate estimation of frequency dependent attenuation and phase velocity is of great importance. Non-parametric methods, such as Fourier analysis, suffers from noise sensitivity, and the variance of the estimated quantities is limited by the signal-to-noise ratio. In this paper we present a parametric method for estimation of these properties. Pulse echo experiments in ethane, oxygen and mixtures of the two show that the proposed method can estimate phase velocity and attenuation with up to 50 times lower variance than standard non-parametric methods.
\end{abstract}

(C) 2006 Elsevier B.V. All rights reserved.

Keywords: System identification; Pulse-echo ultrasound; Phase velocity; Attenuation

\section{Introduction}

When attenuation and speed of sound is estimated using pulse-echo ultrasound, the most common method is standard cross-correlation. In non-dispersive media, the cross-correlation method [1] is the maximum likelihood estimator for both quantities in additive white gaussian noise. In dispersive media, however, the cross-correlation method is biased and frequency dependent estimation methods should be considered.

In this paper a parametric phase velocity and attenuation estimator is proposed. The method is based on a parametrization of the linear dispersion system as a rational transfer function that describes the relationship between two ultrasound echoes, which is then estimated using system identification techniques. In contrast to non-parametric techniques, e.g. Fourier analysis, the proposed estimator method avoids the phase-unwrapping problem since an analytic expression for the continuous phase velocity and attenuation can be derived.

The paper is organized as follows: In Section 2 we describe the theory of wave propagation, parametric and

\footnotetext{
* Corresponding author. Tel.: +46920 493138; fax: +46920492082.

E-mail address: Jesper.Martinsson@1tu.se (J. Martinsson).
}

non-parametric modeling. Section 3 describes the experimental setup and ultrasonic pulse-echo measurements. In Section 4 the methods are evaluated using measurements in ethane, oxygen, and mixtures of the two, followed by a discussion and some concluding remarks.

\section{Theory}

In the first part of this section the acoustic wave propagation model in dispersive media is reviewed. The second part concerns modeling of dispersive wave propagation as linear systems, and the connection to attenuation and phase velocity. The final two parts are dedicated to nonparametric and parametric estimation methods for attenuation and phase velocity.

\subsection{The wave propagation model}

The basic model for acoustic waves in a fluid is obtained using the linearized continuity equation, the linearized force equation, and a relationship between the acoustic pressure and condensation. For linearly dynamic fluids, this relationship is described with a linear system, and a lossy wave equation is obtained 
$\frac{\partial^{2} P(x, \omega)}{\partial x^{2}}=-k^{2}(\omega) P(x, \omega)$,

where $P(x, \omega)$ is the Fourier transform of the acoustic pressure $p(x, t)$ and $k(\omega)$ is the complex frequency dependent wave number. The ODE in Eq. (1) has the solution

$P(x, \omega)=A_{1}(\omega) \mathrm{e}^{-j k(\omega) x}+A_{2}(\omega) \mathrm{e}^{j k(\omega) x}$,

where $j=\sqrt{-1} . A_{1}(\omega)$ and $A_{2}(\omega)$ are to be determined by the boundary conditions, i.e. the amplitude of the wave at $x=0$.

\subsection{Linear system representation}

To find the phase speed and attenuation we have to identify $\exp ( \pm \mathrm{j} k(\omega) x)$ in Eq. (2) and find $A_{1}(\omega)$ and $A_{2}$ $(\omega)$ from the boundary conditions. For a pulse-echo system we can simplify the solution to one wave moving in one direction, assuming near field, perfect reflectivity and short pulses compared to traveling distance. In this case Eq. (2) can be written as

$P(x, \omega)=H(x, \omega) P(0, \omega)$,

where $P(0, \omega)=A_{1}(\omega) \quad$ in Eq. (2) and $H(x, \omega)=$ $\exp -(-\mathrm{j} k(\omega) x)$.

Knowing the acoustic pressure at two different locations say at $x=0$ from the first echo and $x=2 d$ from the second echo, where $d$ is the distance between the transducer and reflector, this can be interpreted as a linear system, see Figs. 1 and 2. In this interpretation $U(\omega)=P(0, \omega)$ is the input signal (Fourier transform of the first echo), $Y(\omega)=P(2 d, \omega)$ is the output signal (Fourier transform of the second echo) and $H(2 d, \omega)$ is the transfer function connecting them.

Using Eq. (2) for $x=2 d$, the attenuation and the phase velocity are defined as

$$
\begin{gathered}
\alpha(\omega)=-\mathfrak{J}\{k(\omega)\}=-\frac{\log |H(2 d, \omega)|}{2 d}, \\
c_{\mathrm{p}}(\omega)=\omega / \mathfrak{R}\{k(\omega)\}=-\frac{2 d \omega}{\arg \{H(2 d, \omega)\}}
\end{gathered}
$$

where $\mathfrak{R}\{\cdot\}$ and $\mathfrak{I}\{\cdot\}$ represents the real- and imaginaryparts, respectively.

From Eq. (4) there are two possible ways to estimate absorption and phase speed. The first one is based on calculating the phase speed $c_{\mathrm{p}}(\omega)$ only from estimates of the absorption $\alpha(\omega)$, using the Kramers-Kronig relationship between the real- and imaginary-parts of $k^{2}(\omega)$, see [2]. This approach is valid under the assumption that the absorption and phase speed are slowly varying functions of frequency.

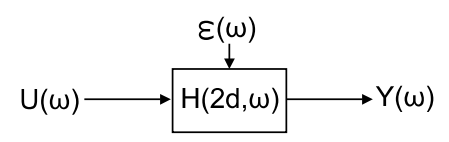

Fig. 1. Linear system representation of the pulse echo system: $U(\omega)$ is the input signal; $Y(\omega)$ is the output signal; $\varepsilon(\omega)$ represents unknown disturbances (measurement noise); $H(2 d, \omega)$ the linear system.
The second approach, investigated in this paper, is to estimate the transfer function $H(2 d, \omega)$ in Eq. (3) and use this estimate together with Eq. (4), without any assumptions made on the dispersion.

\subsection{Non-parametric estimation method}

In this paper we will focus on the Fourier transform (spectral analysis), often used for this type of applications. Using the Fourier transform, the estimate of $H(2 d, \omega)$ is (using $N$ samples)

$$
\hat{H}(\Omega)=\frac{Y_{N}(\Omega)}{U_{N}(\Omega)},
$$

for $\omega=\Omega / T_{\mathrm{s}}$ and $T_{\mathrm{s}}$ is the sampling time. Here $\hat{\imath}$ denotes estimate, and $Y_{N}(\Omega)$ and $U_{N}(\Omega)$ are the Discrete Fourier Transforms (DFT) of the sampled sequences $\{y[n]\}_{1}^{N}$ and $\{u[n]\}_{1}^{N}$.

The accuracy of estimating $H(2 d, \omega)$ in Eq. (3) using Eq. (5) is low and the result is a very erratic function of $\Omega_{l}$, see [3]. If the estimate of $H(2 d, \omega)$ is inaccurate, the attenuation and phase velocity estimates in Eq. (4) will be inaccurate as well, see $[3,4]$.

\subsection{Parametric estimation method}

In general, a parametric method can be described as a mapping from the measured data to the estimated model parameters, $\hat{\boldsymbol{\theta}}$, where $\hat{\boldsymbol{\theta}}=\left[\hat{\theta}_{1}, \hat{\theta}_{2}, \ldots, \hat{\theta}_{q}\right], q$ being the model order.

In this paper, the linear system, $H(2 d, \Omega)$, is modeled as a rational transfer function. The motivation for this choice is primarily its non-complexity in terms of estimation and evaluation, but it will be shown also to be adequate for this application. This choice leads to a model of the form

$$
\begin{aligned}
y[n]= & -a_{1} y[n-1]-\cdots-a_{n a} y[n-n a]+b_{1} u[n-n k] \\
& +\cdots+b_{n b} u[n-n k-n b+1]+\varepsilon[n],
\end{aligned}
$$

where $u[n]=p\left(0, n T_{\mathrm{s}}\right)$ is the sampled first echo (input), $y[n]=p\left(2 d, n T_{\mathrm{s}}\right)$ is the sampled second echo (output of the linear system), and $\varepsilon[n]$ is the residual and representing unmeasured inputs that influence the system (i.e. disturbances and measurement noise).

Given a collection of $N$ samples, $n=1, \ldots, N$, Eq. (6) becomes a system of equations and can be written in matrix notation as

$$
\begin{aligned}
& \mathbf{y}=\boldsymbol{\Phi} \boldsymbol{\theta}+\boldsymbol{\varepsilon} \\
& \boldsymbol{\theta}=\left[a_{1}, \ldots, a_{n a}, b_{1}, \ldots, b_{n b}\right]^{\mathrm{T}} \\
& \boldsymbol{\Phi}=[\boldsymbol{\varphi}[1], \ldots, \boldsymbol{\varphi}[N]]^{\mathrm{T}} \\
& \boldsymbol{\varphi}[n]=[-y[n-1], \ldots,-y[n-n a], \\
& u[n-n k], \ldots, u[n-n k-n b+1]]^{\mathrm{T}},
\end{aligned}
$$

where $\mathbf{y}=[y[1], \ldots, y[N]]^{\mathrm{T}}$ and $\boldsymbol{\varepsilon}=[\varepsilon[1], \ldots, \varepsilon[N]]^{\mathrm{T}}$.

The parameter vector $\theta$ contains the coefficients $a_{i}$ and $b_{i}$ to be estimated. The parameters $n a, n b$ and $n k$ are the structure parameters and describes the characteristics and 
complexity of the model, i.e. its size. There are also other aspects such as complexity, estimate quality, noise modeling and over-fitness to be considered when choosing these parameters, see $[5,6]$.

For $\boldsymbol{\theta} \in R^{q}$ and $N>q$, Eq. (7) is over-determined and the least-squares (LS) estimate is

$\hat{\boldsymbol{\theta}}_{L S}=\left(\boldsymbol{\Phi}^{\mathrm{T}} \boldsymbol{\Phi}\right)^{-1} \boldsymbol{\Phi}^{\mathrm{T}} \mathbf{y}$.

A set of candidate models is obtained, using the LS estimate in Eq. (8) and the two measured echoes (input and output), for different model sizes, i.e. different $n a, n b$ and $n k$. The best model size is determined by cross-validation [7] and residual analysis.

The purpose of the residual analysis is to make sure that the residuals, $\varepsilon[n]$, i.e. the part of the data that the model could not estimate, are zero mean white noise, uncorrelated (or independent) of the input $u[n]$.

Once a proper model is estimated and validated, the estimate of $H(2 d, \omega)$ in Eq. (3) using Eq. (8) is simply

$\hat{H}\left(\Omega, \hat{\boldsymbol{\theta}}_{L S}\right)=\frac{b_{1} \mathrm{e}^{-j \Omega n k}+\cdots+b_{n b} \mathrm{e}^{-j \Omega(n b+n k-1)}}{a_{1} \mathrm{e}^{-j \Omega}+\cdots+a_{n a} \mathrm{e}^{-j \Omega n a}}$,

for $\omega=\Omega / T_{\mathrm{s}}$.

The accuracy of Eq. (9) is high and the variance approaches zero as the sample size grows, in contrast to Eq. (5), see [6].

\subsection{Magnitude and phase response}

The main two advantages for parameterizing the transfer function $H(2 d, \omega)$ in Eq. (4), compared to non-parametric methods, is the increase in accuracy for the estimates and the elimination of the phase unwrapping problem. The first comes from the discussion made in Sections 2.3 and 2.4. The second advantage is given by the parameterized structure itself, enabling calculation of the frequency dependent phase velocity from an analytical expression.

\section{Experimental setup}

For all the experiments in this paper, the ultrasound echoes were measured in a pulse-echo configuration (see Fig. 2). An ultrasound transducer was mounted in a measurement cell, transmitting pulses through the gas towards

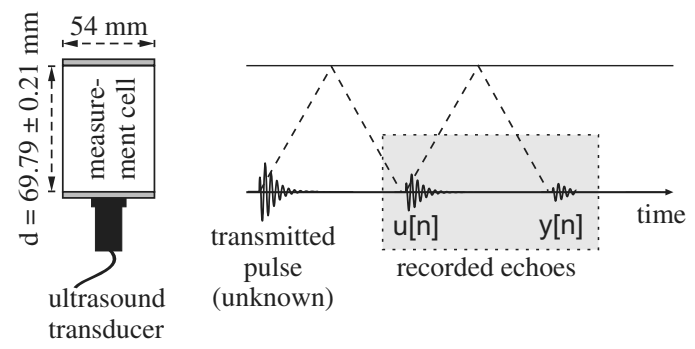

Fig. 2. The measurement cell and the pulse-echo principle. The transducer emits an unknown sound wave. The reflection from the bottom of the measurement cell is then recorded. a stainless steel reflector. The acoustic properties of interest vary with frequency, $f$, and pressure, $P$, and were studied as a function of the ratio $f / P$ [8]. In this work, a $1 \mathrm{MHz}$ air transducer with an effective diameter of $15 \mathrm{~mm}$ was used while the static pressure was varied. For the propagation distances in the present work, diffraction losses can be assumed to be negligible [9].

A custom-built pressure chamber was used to achieve different static pressures. The pressure was varied between 1.54 and 7.4 bar in $12-18$ steps. Since the attenuation in ethane is extremely high at low pressures and high frequencies, we were limited to measurements at higher pressure for ethane (above 1.86 bar).

The pressure in the chamber was measured with an ANDERSON TPP Pressure Transmitter. The transducer was mounted on a stainless steel measurement cell (see Fig. 2), immersed into the pressure chamber. The whole setup was then placed in a temperature controlled chamber (Heraeus Vötsch HT4010). To excite and receive acoustic pulses from the transducer, a Panametrics Pulser/Receiver Model 5072 was used. All pulses were sampled at $100 \mathrm{MHz}$ with an 8-bit Tektronix TDS 724, digitizing oscilloscope. For each measurement, the temperature was recorded using a PT100 sensor.

\section{Results}

\subsection{Parametric model selection and validation}

To make sure that the parametric model is capable of describing the true system, we have to find the best model size using the techniques described in Section 2.

Fig. 3 shows the least-squares cost-function $V(\boldsymbol{\theta})=\boldsymbol{\varepsilon}^{\mathrm{T}} \boldsymbol{\varepsilon}$ evaluated with fresh data, for Ethane at $T=20.6{ }^{\circ} \mathrm{C}$ and $P=2.86$ bar. The best model size was obtained for $q=\operatorname{dim} \theta=14$ and the $=10$ and $n k=9$.

Fig. 4 shows the measured and estimated echoes with corresponding residuals, using the parametric model obtained from Fig. 3. Note that the residual does not show any remaining systematic behavior.

The model was validated by analyzing the residuals in Fig. 4c, and were found to be white and uncorrelated with the input signal, at a $99 \%$ confidence level.

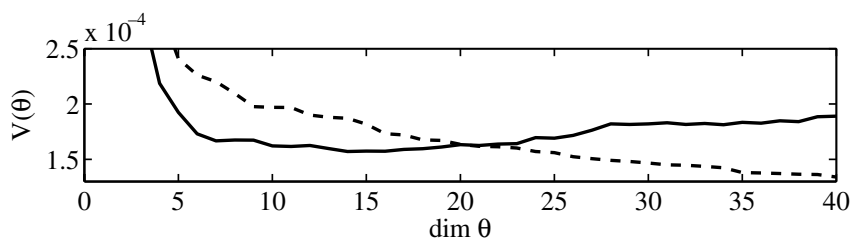

Fig. 3. Cost-functions $V(\boldsymbol{\theta})$ plotted against the model size $q=\operatorname{dim} \boldsymbol{\theta}$, for ethane at $T=20.6{ }^{\circ} \mathrm{C}$ and $P=2.86$ bar. The dashed line represents the cost-function evaluated with calibration data, and the solid line with fresh data (cross-validation). 
(a)

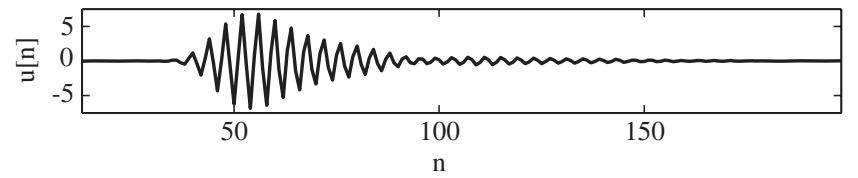

(b)

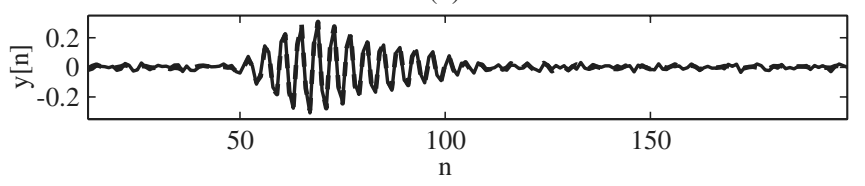

(c)

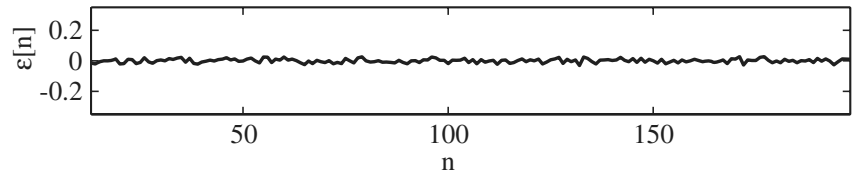

Fig. 4. Measured and estimated echoes with corresponding residuals: (a) measured primary echo; (b) two (coinciding) secondary echoes, solid line the true measured secondary echo, dashed line are estimated from the measured primary echo using the parametric model and (c) the residual (the difference between estimated and measured secondary echo). (a)

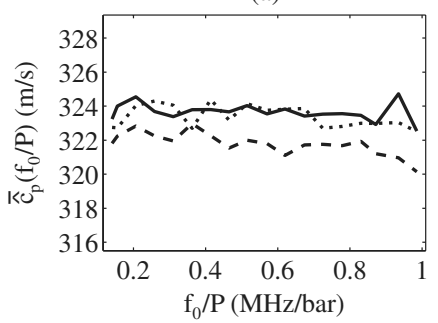

(c)

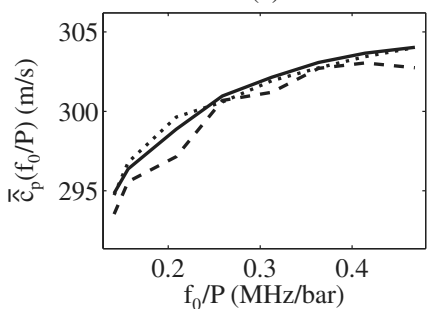

(b)

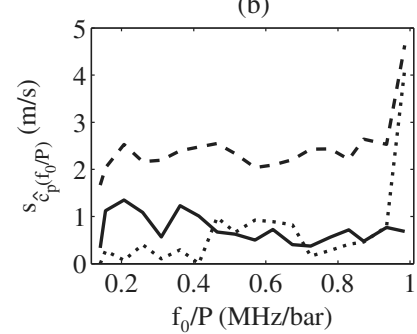

(d)

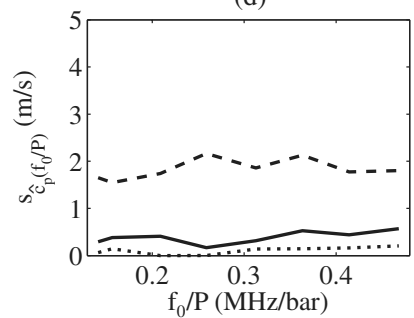

Fig. 5. Mean value $\bar{c}_{\mathrm{p}}$ and estimated standard deviation $s_{\hat{c}_{\mathrm{p}}}$ of 49 phase velocity estimates using the parametric model (solid line), non-parametric (dashed line), and cross-correlation (dotted line): (a,b) Oxygen at $T=20.3{ }^{\circ} \mathrm{C}$ and $(\mathrm{c}, \mathrm{d})$ ethane at $T=20.6{ }^{\circ} \mathrm{C}$. The estimates are evaluated at the center frequency $f 0$ for different pressures.

\subsection{Estimation results}

This section presents a comparison of the non-parametric and parametric estimation of attenuation and phase speed.
Fig. 5 shows phase speed estimates in oxygen and ethane, evaluated at the transducer center-frequency for different pressures.

\section{Conclusions and discussion}

In this paper we have showed how a rational transfer function can be used to describe the wave propagation in dispersive media. The proposed parametric method enables accurate estimation of frequency dependent attenuation and phase velocity with up to 50 times lower variance than the standard non-parametric methods. Robust phase velocity estimation is ensured since the phase unwrapping problem is avoided and replaced by calculation of the phase of an analytical expression.

Cross-correlation cannot be used to estimate $c_{\mathrm{p}}$ in dispersive media, since $c_{\mathrm{p}}$ is frequency dependent. For such media we need to use either parametric or non-parametric spectral methods. In dispersive media the cross-correlation method will give biased results.

\section{Acknowledgement}

This research was performed under grants from The Swedish Energy Agency and the The Kempe Foundation. The authors also like to express their sincerest gratitude towards Dr. Pär-Erik Martinsson for valuable discussions and the experimental data.

\section{References}

[1] A.V. Oppenheim, R.W. Shafer, Discrete-Time Signal Processing, Prentice-Hall, Englewood Cliffs, NJ, 1989.

[2] M. O’Donnell, E.T. Jayes, J.G. Miller, Kramers-Kronig relationship between ultrasonic attenuation and phase velocity, J. Acoust. Soc. Am. 69 (3) (1981) 696-701.

[3] D.R. Brillinger, Time Series: Data Analysis and Theory, HoldenDay series in time series analysis, Holden-Day, San Francisco, 1981.

[4] L. Ljung, On the estimation of transfer functions, Automatica 21 (6) (1985) 677-696.

[5] T. Söderström, P. Stoica, System Identification, Prentice-Hall, Englewood Cliffs, NJ, 1989.

[6] L. Ljung, System Identification: Theory for the User, 2nd ed., PrenticeHall, Englewood Cliffs, NJ, 1987.

[7] J. Shao, Linear model order selection by cross-validation, J. Am. Stat. Assoc. 88 (June) (1993) 486-494.

[8] A.B. Bhatia, Ultrasonic Absorption - An Introduction to the Theory of Sound Absorption and Dispersion in Gases, Liquids and Solids, Dover Publications, New York, 1985.

[9] G.S. Kino, Acoustic Waves: Devices, Imaging, and Analog Signal Processing, Prentice-Hall, Englewood Cliffs, NJ, 1987. 